FACTA UNIVERSITATIS

Series: Mechanical Engineering Vol. 16, N² 2, 2018, pp. 157 - 170

https://doi.org/10.22190/FUME180404015J

Original scientific paper

\title{
A NUMERICAL AND EXPERIMENTAL ANALYSIS OF THE DYNAMIC STABILITY OF HYDRAULIC EXCAVATORS
}

\author{
UDC 621
}

\author{
Dragoslav Janošević, Jovan Pavlović, Vesna Jovanović, Goran Petrović \\ University of Niš, Faculty of Mechanical Engineering, Serbia
}

\begin{abstract}
The paper presents the results of a numerical and experimental analysis of the dynamic stability of hydraulic excavators. The analysis has employed the software developed on the basis of a defined general dynamic mathematical model of an excavator grounded in Newton-Euler equations as well as the measured quantities of the excavator operating state in exploitation conditions. The defined model is used to model the members of the excavator kinematic chain using rigid bodies while the actuators (hydraulic cylinders and hydraulic motors) of the excavator drive mechanisms are modeled with elastically dampened elements. The elastically dampened characteristics of the actuators are defined with regard to the size of the actuator as well as to the compressibility and temperature of the hydraulic oil used in the excavator hydrostatic drive system. To illustrate the analysis, the paper provides the results of the analysis of the dynamic stability of a $16000 \mathrm{~kg}$ tracked excavator equipped with a manipulator digging bucket of $0.6 \mathrm{~m}^{3}$ in capacity.
\end{abstract}

Key Words: Hydraulic Excavators, Dynamic Stability, Oil Temperature, Modeling

\section{INTRODUCTION}

In modern hydraulic excavators one of the most important parameters is the indicator of the excavator's stable operation. International standards provide the conditions for examination and determination of the static indicators of the excavator stability and hydraulic stability of the excavator drive mechanisms (SAE J1097). Previous research in the area has been related to the development of mathematical models for the analysis of the static stability of hydraulic excavators [1-4]. However, the studies have shown that hydraulic excavators (and other hydraulic mobile machines) act like dynamic oscillating

Received April 04, 2018 / Accepted May 15, 2018

Corresponding author: Dragoslav Janošević

University of Niš, Faculty of Mechanical Engineering, Department for Material Handling Systems and Logistics,

A. Medvedeva 14, 18000 Niš, Serbia

E-mail: janos@masfak.ni.ac.rs 
systems during their cyclic operation [5-7] where actuators (hydraulic motors and hydraulic cylinders) of the excavator drive mechanisms appear as extremely elastically dampened system members in the shape of hydraulic springs. They occur due to the compressibility of oil in the actuator displacement and ducts of the excavator hydrostatic drive system. The dynamic behavior of an excavator is pronounced during the material transfer and unloading operations when disturbances which may cause the dynamic instability of the excavator occur. Elastically dampened characteristics of the excavator actuators, therefore, the dynamic stability of the excavator itself, are greatly influenced by the temperature of the hydraulic oil in the excavator drive system.

The paper contains an analysis of the influence that the oil temperature in the excavator hydrostatic drive system exerts on the excavator's dynamic stability.

\section{DYNAMIC EXCAVATOR MODEL}

When analyzing the dynamic stability of an excavator one observes the physical model of the machine with the kinematic chain of general configuration comprising: support and movement member $L_{1}$ (Fig. 1a), rotating member $L_{2}$, boom $L_{3}$, stick $L_{4}$ and bucket $L_{5}$. The members of the excavator kinematic chain form the rotating kinematic pairs of the fifth class. The members of the kinematic pairs are joined, directly or indirectly, by hydraulic actuators: hydraulic motor $C_{2}$ (Fig. 1b) of the platform rotation drive and hydraulic cylinders $C_{3}, C_{4}, C_{5}$ of the manipulator drive which are powered, through the hydraulic distributor 3 , by hydraulic pumps 2 driven by the diesel engine 1 .

With regard to the physical model, a dynamic mathematical model of the excavator (Fig. 2) was adopted with the following assumptions: a) the mechanical model of the excavator is a non-conservative system with stationary and ideal links, b) small system oscillations are observed around the stable balance position, c) the excavator support base has elastically dampened properties, d) the members of the excavator kinematic chain are rigid bodies, e) the hydraulic actuators of the drive mechanisms are elastically dampened systems due to the oil compressibility and viscosity, and f) the bulk modulus of the hydraulic oil is constant for a certain oil pressure and temperature.

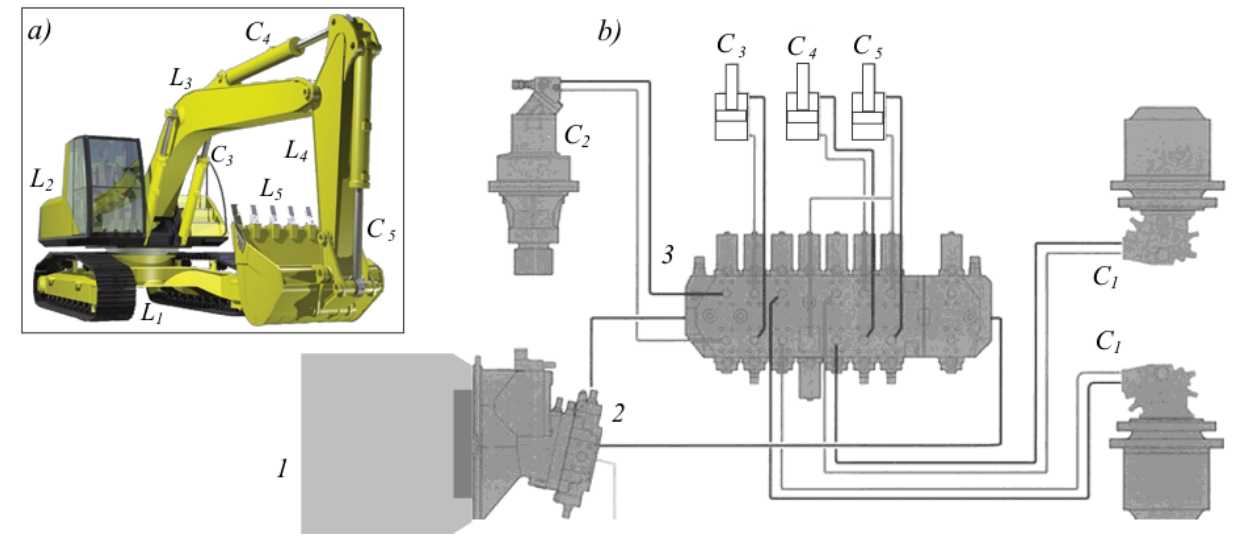

Fig. 1 Hydraulic excavators: a) physical model, b) hydrostatic drive system 
In the mathematical model, a member of excavator kinematic chain $L_{i}$ is defined, in its local coordinate system $O_{i} x_{i} y_{i} z_{i}$, by a set of geometric, kinematic and dynamic parameters (Fig. 2):

$$
L_{i}=\left\{\mathbf{s}_{i}, \mathbf{t}_{i}, m_{i}, \mathbf{J}_{i}\right\}
$$

where: $s_{i}$ - the vector of the centre position of joint $O_{i+1}$ which links chain member $L_{i}$ to next member $L_{i-1}$ (vector magnitude $\boldsymbol{s}_{i}$ represents the kinematic length of the member), $\boldsymbol{t}_{i}-$ the vector of the mass centre position of member $L_{i}, m_{i}$ - the member mass, $\boldsymbol{J}_{i}-$ the moment of the member inertia.

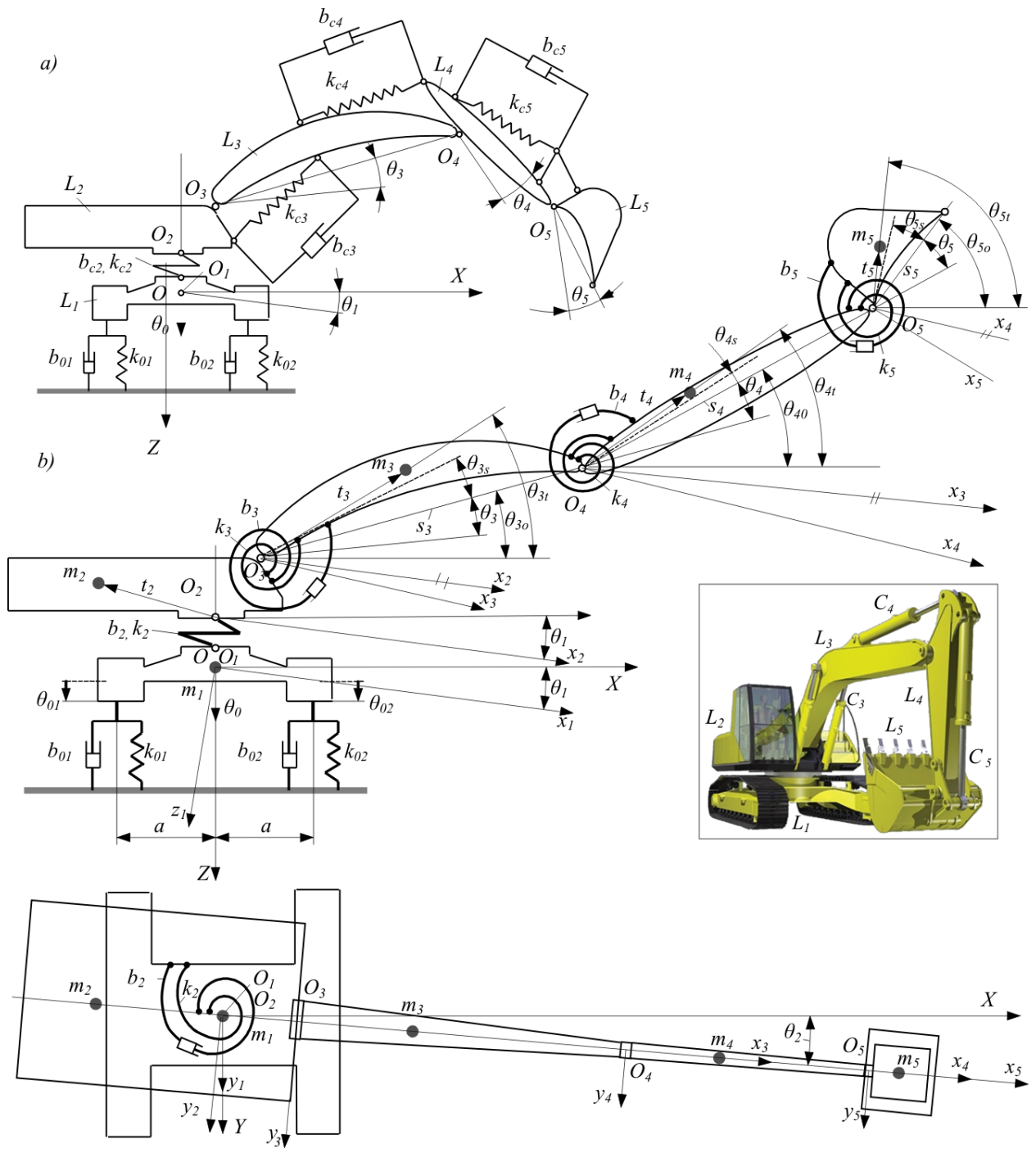

Fig. 2 Mathematical model of the excavator 
In the mathematical model, the parameters of the actuators (hydraulic motors and hydraulic cylinders) of the excavator drive mechanisms are determined by a set of quantities:

$$
C_{i}=\left\{d_{i 1}, d_{i 2}, c_{i p}, c_{i k}, m_{c i}, n_{c i}\right\}
$$

where: $d_{i 1}, d_{i 2}$ - the parameters of actuator sizes (minimal and maximal specific flow of hydraulic motors, i.e. the piston diameter and the piston rod diameter in the hydraulic cylinder), $c_{i p}$ - the minimal length of the hydraulic cylinder when the piston rod is fully drawn in, $c_{i k}$ - the maximal length of the hydraulic cylinder when the piston rod is fully drawn out, $m_{c i}$ - the actuator mass, $n_{c i}$ - the number of drive mechanism actuators.

The movement of the adopted dynamic mathematical model of the excavator is defined by a set of generalized coordinates (Fig. 2):

$$
\Theta=\left\{\theta_{0}, \theta_{1}, \theta_{2}, \theta_{3}, \theta_{4}, \theta_{5}\right\}
$$

where: $\theta_{0}$ - the vertical movement of the mass centre of the support and movement member, $\theta_{1}$ - the angle of rotation around the main longitudinal central axis of inertia $O_{1} y_{1}$ of the support and movement member, $\theta_{2}$ - the angle of rotation of the rotating member around the $\mathrm{O}_{2} z_{2}$ axis of the axial bearing, $\theta_{3}$ - the angle of rotation of the manipulator boom around the $\mathrm{O}_{3} y_{3}$ axis of the joint to which the rotating member is connected, $\theta_{4}-$ the angle of rotation of the manipulator stick around the $O_{4} y_{4}$ axis of the joint at the end of the boom, $\theta_{5}-$ the angle of rotation of the manipulator bucket around the $O_{5} y_{5}$ axis of the joint at the end of the stick.

The generalized coordinates are assumed to be small quantities measured from the position of the system's stable balance. Critical positions of the excavator are analyzed when the manipulator plane is perpendicular to the plane of the tracked support and movement member.

The position of the excavator is defined in relation to the immovable (absolute) coordinate system $O X Y Z$. Coordinate beginning $O$ of the absolute system is in the centre of the support and movement member mass, while the $O X$ axis is directed towards the main transverse central axis of inertia of the same member in the position of the static balance of the entire system.

The vector of position $\boldsymbol{r}_{t i}$ of the mass centre of member $L_{i}$ of the excavator kinematic chain is determined in relation to the absolute coordinate system with the following equation:

$$
\boldsymbol{r}_{t i}=\boldsymbol{s}_{o}+\sum_{j=1}^{i-1} A_{o j} \boldsymbol{s}_{j}+A_{o i} \cdot t_{i}
$$

where: $s_{0}=\left[\begin{array}{lll}0 & 0 & z_{c}\end{array}\right]^{\mathrm{T}}-$ the vector of deformation of the excavator model support base, i.e. the vector of displacement of the mass centre of the support and movement member in relation to the absolute coordinate system, $\boldsymbol{s}_{j}$ - the vector of the position of joint $O_{j}$ centre in relation to previous joint $O_{j-1}$, determined in the local coordinate system, $\boldsymbol{t}_{i}-$ the vector of the position of member $L_{i}$ mass centre in relation to joint $O_{i}$ centre, $A_{o j}$ - the transitional vector matrix from local coordinate system $O_{j} x_{j} y_{j} z_{j}$ to the absolute coordinate system [7, 8]. 


\section{DIFFERENTIAL EQUATIONS OF MOTION}

Differential equations of motion of the defined mathematical model of the excavator are determined by using Lagrange's equations of the second kind:

$$
\frac{d}{d t} \frac{\partial E_{k}}{\partial \dot{\Theta}_{i}}-\frac{\partial E_{p}}{\partial \Theta_{i}}+\frac{\partial \Phi}{\partial \dot{\Theta}_{i}}=0
$$

where: $E_{k}, E_{p}, \Phi-$ the kinetic and potential energy and function of the system dissipation, respectively [9].

\subsection{Kinetic energy}

For the established dynamic model of the excavator, the kinetic energy of the system is expressed using the equation:

$$
\begin{aligned}
& 2 E_{k}=m_{1} v_{t 1}^{2}+J_{1 y} \dot{\theta}_{1}^{2}+m_{2} v_{t 2}^{2}+J_{2 y} \dot{\theta}_{1}{ }^{2}+J_{2 z} \dot{\theta}_{2}{ }^{2}+ \\
& +m_{3} v_{t 3}^{2}+J_{3 y} \dot{\theta}_{3}^{2}+m_{4} v_{t 4}^{2}+J_{4 y} \dot{\theta}_{4}^{2}+m_{5} v_{t 5}^{2}+J_{5 y} \dot{\theta}_{5}^{2}
\end{aligned}
$$

where: $m_{i}$ - the mass of an excavator kinematic chain member, $(i=1, \ldots, 5), v_{t i}-$ the absolute velocities of the mass centers of the excavator kinematic chain members, $J_{i y}, J_{i z}-$ the appropriate main central axial moments of inertia of the masses of the excavator kinematic chain members. Differentiating equation (4), in the absolute coordinate system, yields the vector of velocity $v_{t i}$ of the mass centers of the excavator kinematic chain members in the following form:

$$
\boldsymbol{v}_{t i}=\dot{\boldsymbol{s}}_{o}+\sum_{j=1}^{i-1} \dot{A}_{o j} \boldsymbol{s}_{j}+\sum_{j=1}^{i-1} A_{o j} \dot{\boldsymbol{s}}_{j}+\dot{A}_{o i} \boldsymbol{t}_{i}+A_{o i} \dot{\boldsymbol{t}}_{i}
$$

where: $\dot{\boldsymbol{s}}_{o}, \dot{\boldsymbol{s}}_{j}, \dot{\boldsymbol{t}}_{i}, \dot{A}_{o i}, \dot{A}_{o j}-$ the derivatives of the appropriate vectors of position and transitional matrix systems, where, along the assumptions of small quantities of generalized coordinates $\theta_{i}$, the approximate values $\cos \theta_{i} \approx 1$ and $\sin \theta_{i} \approx \theta_{i}$ are introduced, thus linearizing the elements of transitional matrices $A_{i j}$.

\subsection{Potential energy}

For the established dynamic model of the excavator, the potential energy of the system is determined using the equation:

$$
\begin{aligned}
& 2 E_{p}=k_{o}\left(\theta_{o 1}+a \theta_{1}+\theta_{o}\right)^{2}-k_{o} \theta_{o 1}^{2}+k_{o}\left(\theta_{o 2}-a \theta_{1}+\theta_{o}\right)^{2}-k_{o} \theta_{o 2}^{2}+k_{2} \theta_{2}^{2}+k_{3}\left(\theta_{3 s}+\theta_{3}\right)^{2}- \\
& -k_{3} \theta_{3 s}^{2}+k_{4}\left(\theta_{4 s}+\theta_{4}\right)^{2}-k_{4} \theta_{4 s}^{2}+k_{5}\left(\theta_{5_{s}}+\theta_{5}\right)^{2}-k_{5} \theta_{5 s}^{2}-2 g \sum_{i=1}^{5} m_{i}\left(z_{i}-z_{o i}\right)
\end{aligned}
$$

where: $z_{i}, z_{o i}$ - the current and initial coordinates of the positions of mass centers of the excavator hydraulic chain members in relation to the absolute coordinate system, $k_{o}-$ the stiffness of the elastic support base of the excavator, $\theta_{o 1}, \theta_{o 2}-$ the static deflections of the 
elastic base beneath the support and movement member of the excavator, $k_{3}, k_{4}, k_{5}-$ the torsional stiffnesses of springs equivalent to the stiffnesses of the elastic hydraulic actuators of drive mechanisms of the manipulator boom, stick and bucket, $\theta_{3 s}, \theta_{4 s}, \theta_{5 s}-$ the angles of static deflections of torsion springs of the manipulator boom, stick and bucket.

By neglecting the influence of the generalized coordinate of platform $\theta_{2}$ rotation, as a small quantity, and using the approximate equivalences:

$$
\sin \theta \approx \theta ; \quad 1-\cos \theta \approx \sin \theta \approx \theta ; \quad 1-\cos \theta \approx \frac{\theta^{2}}{2} \quad \forall i=1,2,3
$$

The elastic action of an actuator with stiffness $k_{c i}$ (Fig. 2a), in relation to the joint of the drive mechanism which it powers, is substituted by an equivalent action of torsion springs (Fig. 2b), whose stiffness $k_{i}$ is determined by the following equation:

$$
k_{i}=i_{c i}^{2} \cdot \mathrm{k}_{\mathrm{ci}} \quad \forall i=2,3,4,5
$$

where: $i_{c i}$ - the transitional function of the excavator drive mechanism moment [6].

The base (ground) stiffness beneath the support and movement mechanism of the excavator is determined using the equation [9]:

$$
k_{o}=E_{r o} \cdot A_{1}
$$

where: $E_{r o}$ - the ground reaction modulus, $A_{l}$ - the footprint surface area of the support and movement member of the excavator.

Actuator displacements and hydraulic ducts in the drive mechanisms are filled with compressible hydraulic oil of the excavator hydrostatic drive system. Due to the hydraulic oil compressibility, the actuators are modeled using springs with an appropriate stiffness $k_{c i}$, determined with the equation [10]:

$$
k_{c i}=\left\{\begin{array}{lc}
\frac{A_{i 1}^{2} E_{u}}{\left[A_{i 1}\left(c_{i}-c_{i p}\right)+V_{i 1}\right]}+\frac{A_{i 2}^{2} E_{u}}{\left[A_{i 2}\left(c_{i k}-c_{i}\right)+V_{i 2}\right]} & \forall \theta_{i}=3,4,5 \text {-for hydraulic cylinders } \\
\frac{2 \cdot d_{21}^{2} E_{u}}{V_{21}} ; V_{21}=V_{22} & \text {-for hydraulic motors }
\end{array}\right.
$$

where: $A_{i 1}, A_{i 2}$ - the operating surface areas of the hydraulic cylinder on the piston and piston rod end, $E_{u}$ - the elasticity (bulk) modulus of the hydraulic oil, $c_{i}, c_{i p}, c_{i k}-$ the current, initial and final length of the hydraulic cylinder, $d_{2 I}$ - the maximal specific flow (displacement) of the hydraulic motor, $V_{i 2}-$ the displacement of the actuator hydraulic ducts.

\subsection{Dissipation function}

For the established dynamic model of the excavator, the potential energy of the system is determined using the equation:

$$
2 \Phi=b_{o}\left(\dot{\theta}_{o}+a \cdot \dot{\theta}_{1}\right)^{2}+b_{o}\left(\dot{\theta}_{o}-a \cdot \dot{\theta}_{1}\right)^{2}+b_{2} \dot{\theta}_{2}^{2}+b_{3} \dot{\theta}_{3}^{2}+b_{4} \dot{\theta}_{4}^{2}+b_{5} \dot{\theta}_{5}^{2}
$$


where: $b_{o}$ - the damping coefficient of the excavator support base, $b_{i}$ - the damping coefficient of the hydraulic actuators of drive mechanisms of the platform, boom, stick and bucket, reduced to the joints of the excavator kinematic chain powered by them.

The damping coefficient of the excavator support base is determined by the following equation [11]:

$$
b_{o}=2 \cdot k_{o} \cdot E_{p o}
$$

where: $k_{o}$ - the stiffness of the ground, $E_{p o}-$ the damping modulus of the ground.

The damping coefficient of the hydraulic actuators of drive mechanisms is determined by the following expression:

$$
b_{i}=i_{c i}^{2} \cdot b_{c \mathrm{i}} \quad \forall \quad i=2,3,4,5
$$

where the damping coefficient of hydraulic cylinder $b_{c i}$ is defined by the following equation [11]:

$$
b_{c i}=\frac{\pi \cdot \eta_{u} \cdot l_{i} \cdot d_{i 1}^{2}}{\left(D_{i}-d_{i 1}\right)^{2}}\left[3+\frac{3}{4} \frac{d_{i 1}}{\left(D_{i}-d_{i 1}\right)}\right] \quad \forall i=3,4,5
$$

where: $\eta_{u}$ - the dynamic viscosity of hydraulic oil, $d_{i l}$ - the piston diameter, $l_{i}-$ the piston length, and $D_{i}$ - the internal diameter of the hydraulic cylinder.

Substituting the expression for kinetic (6) and potential (8) energy, and the expression for the dissipation function (13) into Lagrange's equations of the second kind (5), one obtains a system of six homogenous differential equations of the oscillating system:

$$
\boldsymbol{M} \ddot{\Theta}+\boldsymbol{F} \dot{\Theta}+\boldsymbol{K} \Theta=0
$$

where: $\boldsymbol{M}$ - the inertial matrix, $\boldsymbol{F}$ - the matrix of resistance forces, $\boldsymbol{K}$ - the stiffness matrix.

The equations of motion of the excavator dynamic system are determined using the method of addition of the main forms of oscillation with the next equation

$$
\Theta=V \cdot \boldsymbol{u}
$$

where: $V$ - modal matrix of eigenvectors, $\boldsymbol{u}$ - vector of normal coordinates [12].

\section{EXAMPLE}

As an example, the numerical and experimental analysis of dynamic stability was performed on a $16000 \mathrm{~kg}$ tracked excavator equipped with a manipulator digging bucket of $0.6 \mathrm{~m}^{3}$ in capacity.

\subsection{Numerical analysis}

Using the developed programs, as an example, the analysis was performed on the influence of oil temperature of the drive system on the dynamic stability of a tracked excavator with the mass of $16000 \mathrm{~kg}$, equipped with a manipulator with the bucket capacity 
of $0.6 \mathrm{~m}^{3}$. The following files are input at the start of the program: the excavator kinematic chain file (with geometric and dynamic parameters of each member), the drive mechanisms file, the support base characteristics, and the characteristics of the oil in the hydrostatic drive system of the excavator. The program output yields the generalized system coordinates in the function of time.

The numerical analysis monitored the change in the generalized coordinates (Fig. 2):

$$
\Theta_{i}=\theta_{o}, \theta_{1}, \theta_{3}, \theta_{4}
$$

The set system conditions and parameters are given in Table T1. The position of the excavator was observed during the unloading of the material when, according to the excavator measurements in exploitation conditions, primary movements of the support and movement member and the manipulation boom appeared $[11,13]$.

Table 1 System conditions and parameters

Initial position coordinates of the support and movement mechanism and the platform

$\theta_{o}=0^{\circ}, \theta_{1}=0^{\circ}, \theta_{2}=0^{\circ}$

Initial position coordinates of the manipulator kinematic chain

$\theta_{3 o}=35^{\circ}, \theta_{4 o}=0^{\circ}, \theta_{5 o}=-120^{\circ}$

Bulk modulus of hydraulic oil at $80^{\circ} \mathrm{C}$

$E_{u}=1.4 \cdot 10^{9}\left[\mathrm{~N} / \mathrm{m}^{2}\right]$

Bulk modulus of hydraulic oil at $90^{\circ} \mathrm{C}$

$E_{u}=1.2 \cdot 10^{9}\left[\mathrm{~N} / \mathrm{m}^{2}\right]$

Modulus of reaction of the excavator support base

$E_{r o}=5.5 \cdot 10^{8}\left[\mathrm{~N} / \mathrm{m}^{3}\right]$

Damping modulus of the excavator support base

$E_{p o}=0.005[s]$

Initial boom angle velocity

$\dot{\theta}_{3 o}=-2[\mathrm{rad} / \mathrm{s}]$

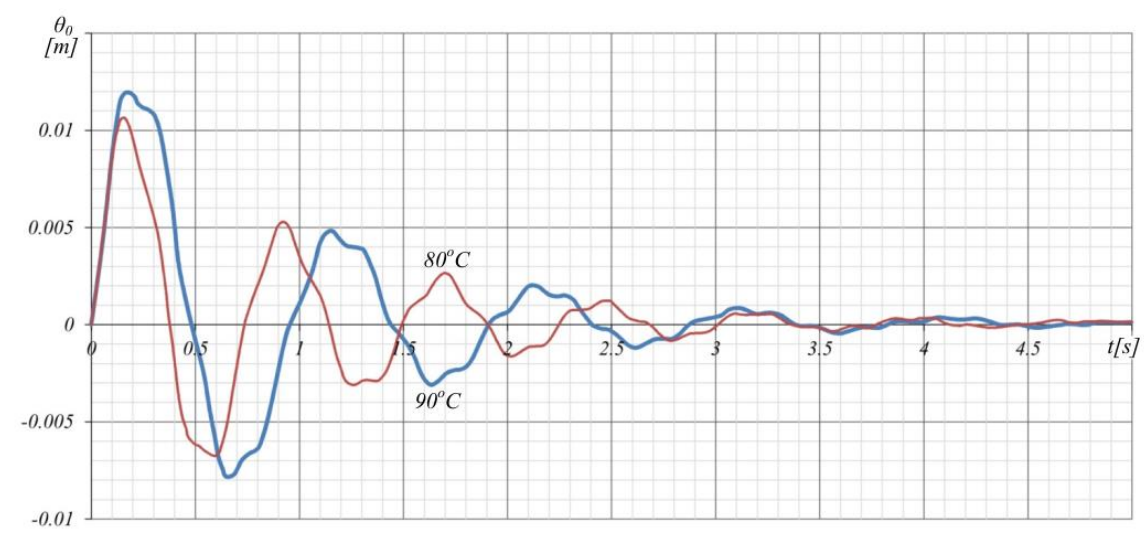

Fig. 3 Changes in generalized coordinates $\theta_{0}$ of the support and movement member for the initial position of the manipulator and at different oil temperatures 

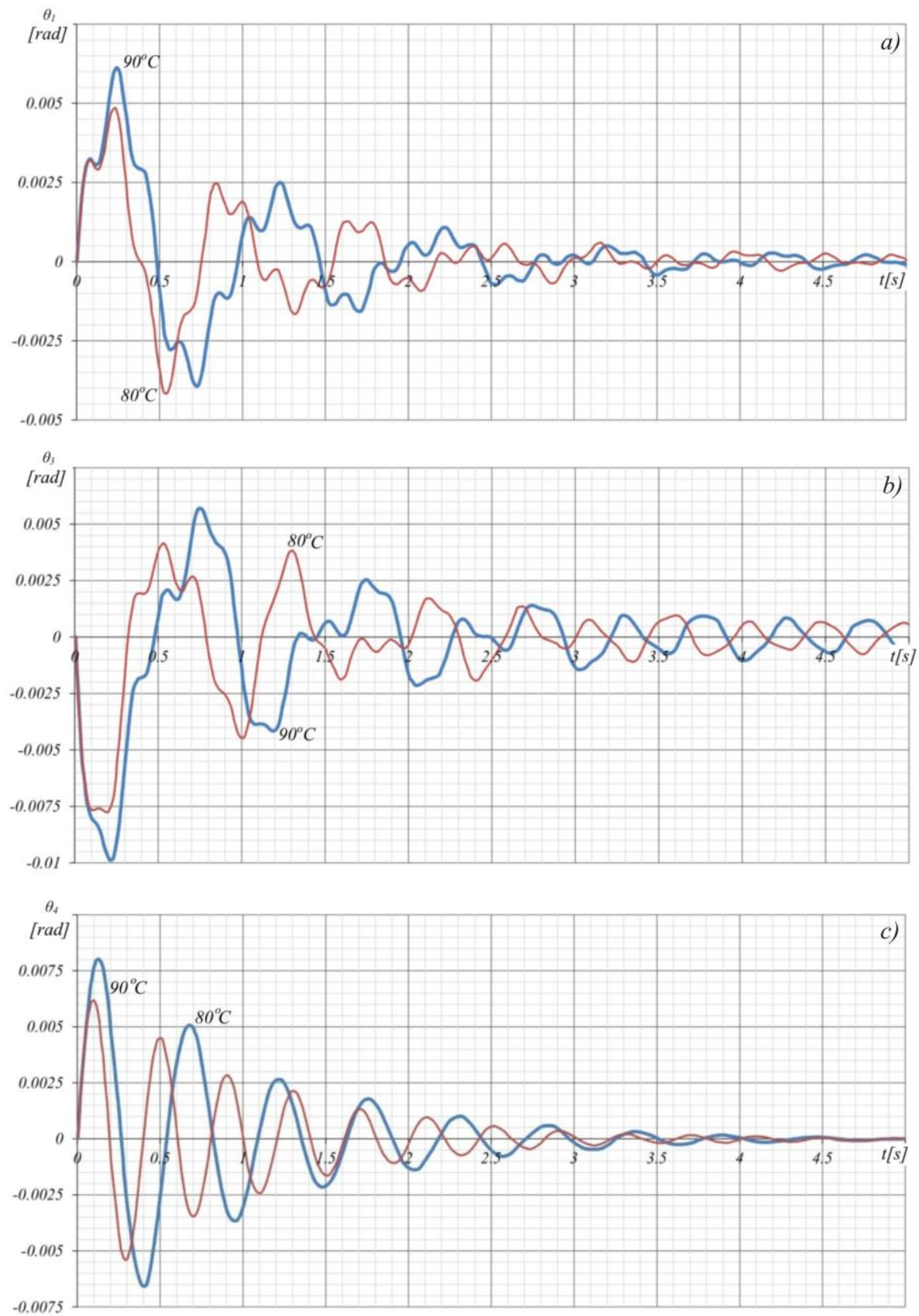

Fig. 4 Changes in the generalized coordinates for the initial position of the manipulator and at different oil temperatures: a) $\theta_{1}$ of the support and movement member,

b) $\theta_{3}$ of the boom angle, c) $\theta_{4}$ of the stick angle 
Based on certain matrices and the given initial conditions of movement, and using the developed program, the differential equations were solved and the changes in the generalized coordinates of free oscillations of the excavator were obtained (Figs. 3 and 4).

Diagrams of the vertical $\theta_{\mathrm{o}}$ (Fig. 3) and angular $\theta_{1}$ displacement (Fig. 4a) of the support and movement member and diagrams of the change in the generalized coordinate of the boom $\theta_{3}$ (Fig. 4b) and stick $\theta_{4}$ (Fig. 4c) show that the oscillatory- dampened movement of the excavator kinematic chain members with different amplitudes and oscillation periods for different temperatures of the hydraulic oil in the excavator drive system.

It is noticeable that at the higher oil temperature $90^{\circ} \mathrm{C}$, when the dynamic viscosity and modulus of elasticity are smaller, amplitudes and periods of oscillation of the movement mechanism are greater in relation to the lower oil temperature $80^{\circ} \mathrm{C}$. Furthermore, changes in the generalized coordinates of boom $\theta_{3}$ (Fig. 4b) and stick $\theta_{4}$ (Fig. 4c) possess a similar oscillatory-dampened character. It is characteristic that the damping time of oscillations of all generalized coordinates is approximately the same and it amounts to 4 s. Only the generalized boom coordinate, whose initial movement conditions cause the disturbance in the dynamic system of the excavator, has a longer damping period.

\subsection{Experimental analysis}

The experimental analysis of the dynamic stability of the excavator was performed on the basis of the changes in measured quantities in exploitation conditions. During the excavator testing, the following quantities of the excavator exploitation working condition were measured: the vertical displacement of support and movement member $c_{1}$, the angle of platform rotation $c_{2}$, the kinematic length of the hydraulic cylinders of boom $c_{3}$, stick $c_{4}$ and bucket $c_{5}$ and the pressure in the actuators of the excavator drive mechanisms.

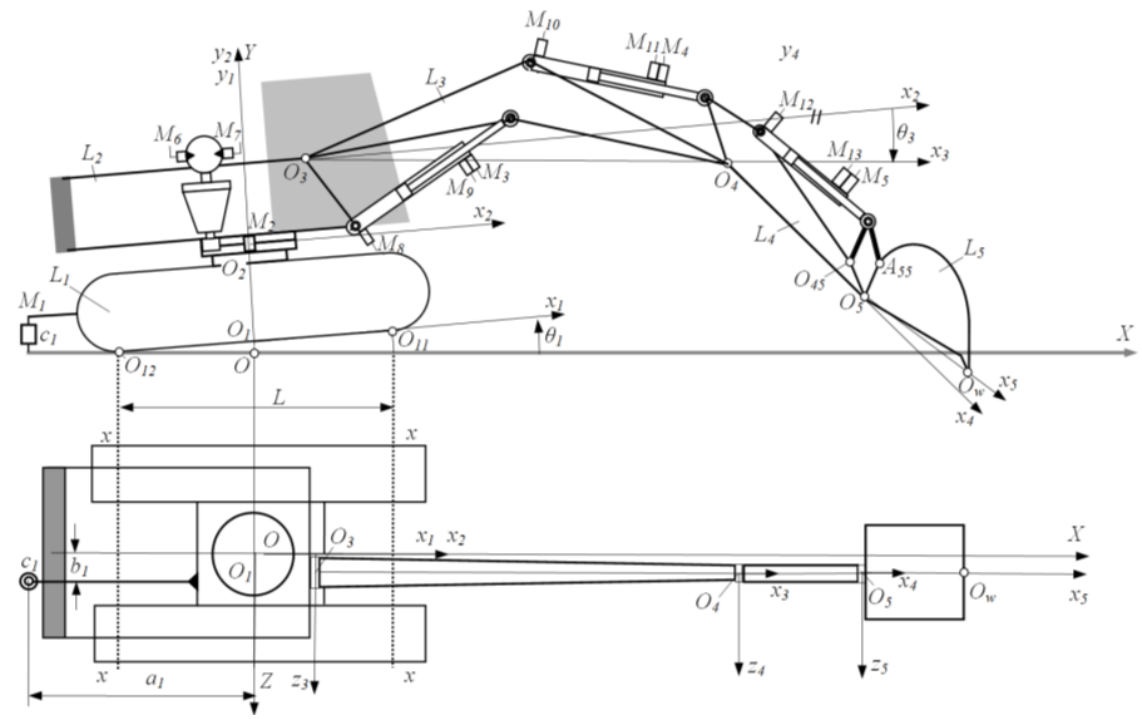

Fig. 5 Mathematical model of the excavator for the experimental analysis 
On the basis of the measured quantities of the excavator operating state, and by using the developed program, the change in the generalized coordinates $\left(\theta_{0}, \theta_{1}, \theta_{3}\right)$ of the positions of the excavator kinematic chain members was determined during various manipulation tasks of the excavator in exploitation conditions (Fig. 5).

Table 2 Measured quantities in exploitation conditions

\begin{tabular}{|c|c|c|c|}
\hline iensors & Measured quantities & Index & Dim \\
\hline M1 & The vertical displacement of the support and movement member & $c_{1}$ & $\mathrm{~m}$ \\
\hline$M 2$ & The angle of the platform rotation & $c_{2}$ & $\mathrm{rad}$ \\
\hline$M 3$ & The stroke of the boom hydraulic cylinder & $c_{3}$ & $\mathrm{~m}$ \\
\hline M4 & The stroke of the stick hydraulic cylinder & $c_{4}$ & $\mathrm{~m}$ \\
\hline M5 & The stroke of the bucket hydraulic cylinder & $c_{5}$ & $\mathrm{~m}$ \\
\hline M6 & $\begin{array}{l}\text { The pressure in the one duct of the hydraulic motor for platform } \\
\text { rotation drive }\end{array}$ & $p_{21}$ & $\mathrm{MPa}$ \\
\hline M7 & $\begin{array}{l}\text { The pressure in the another duct of the hydraulic motor for platform } \\
\text { rotation drive }\end{array}$ & $p_{22}$ & $\mathrm{MPa}$ \\
\hline$M 8$ & The pressure in the boom hydraulic cylinder on the piston end & $p_{31}$ & $\mathrm{MPa}$ \\
\hline$M 9$ & The pressure in the boom hydraulic cylinder on the piston rod end & $p_{32}$ & $\mathrm{MPa}$ \\
\hline$M 10$ & The pressure in the stick hydraulic cylinder on the piston end & $p_{41}$ & $\mathrm{MPa}$ \\
\hline$M 11$ & The pressure in the stick hydraulic cylinder on the piston rod end & $p_{42}$ & $\mathrm{MPa}$ \\
\hline$M 12$ & The pressure in the bucket hydraulic cylinder on the piston end & $p_{51}$ & $\mathrm{MPa}$ \\
\hline$M 13$ & The pressure in the bucket hydraulic cylinder on the piston rod end & $p_{52}$ & $\mathrm{MPa}$ \\
\hline
\end{tabular}

Generalized coordinates $\theta_{1}$ of the support and movement member of tested excavator are determined by the following equation (Fig. 5):

$$
\theta_{1}=\left\{\begin{array}{ll}
-\operatorname{arctg} \frac{2 \cdot c_{1}}{L+2 \cdot a_{1}} & \forall c_{1} \geq 0 \\
-\operatorname{arctg} \frac{2 \cdot c_{1}}{2 \cdot a_{1}-L} & \forall c_{1} \leq 0
\end{array} \theta_{1}= \begin{cases}-\operatorname{arctg} \frac{2 \cdot c_{1}}{L+2 \cdot a_{1}} & \forall c_{1} \geq 0 \\
-\operatorname{arctg} \frac{2 \cdot c_{1}}{2 \cdot a_{1}-L} & \forall c_{1} \leq 0\end{cases}\right.
$$

where: $a_{1}-$ the coordinate of the sensor position, $L-$ the length of the excavator track footprint.

The changes in the following generalized coordinates of the excavator kinematic chain members are selected and presented here: $\theta_{0}$ - the vertical displacement of the centre of mass of the support and movement member (Fig. 6), $\theta_{1}$ - the angle of displacement of the support and movement member (Fig. 7), $\theta_{3}$ - the angle of the rotation of the manipulator boom (Fig. 8).

The results yielded by the experimental dynamic analysis of the excavator show that the hydraulic excavators act like very sensitive dynamic oscillatory systems where as elastically dampened elements of the system occur hydrostatic actuators (hydraulic motors and hydraulic cylinders) of excavator drive mechanisms, due to hydraulic oil compressibility.

Diagrams of the changes in generalized coordinates $\theta_{0}$ (Fig. 6), $\theta_{1}$ (Fig. 7), $\theta_{3}$ (Fig. 8) show that a pronounced oscillatory state of the excavator occurs at the end of the digging operation, when the digging resistance drops rapidly, and it appears as a force impulse 
acting on the excavator's elastically dampened system. The oscillatory displacement also occurs at the beginning of the material moving operation when the boom begins to move. However, primary oscillatory displacement of the member of the support and movement mechanism kinematic chain appear during the material unloading operation at the moment when the bucket is being emptied and when the system dynamic parameters - the mass and the moment of inertia of the scooped material - change rapidly.

The results of the experimental dynamic analysis of the excavator stability show the oscillatory-dampened character of the generalized coordinates with the damping time of around $4 \mathrm{~s}$, which approximately corresponds to the time obtained in the numerical analysis.

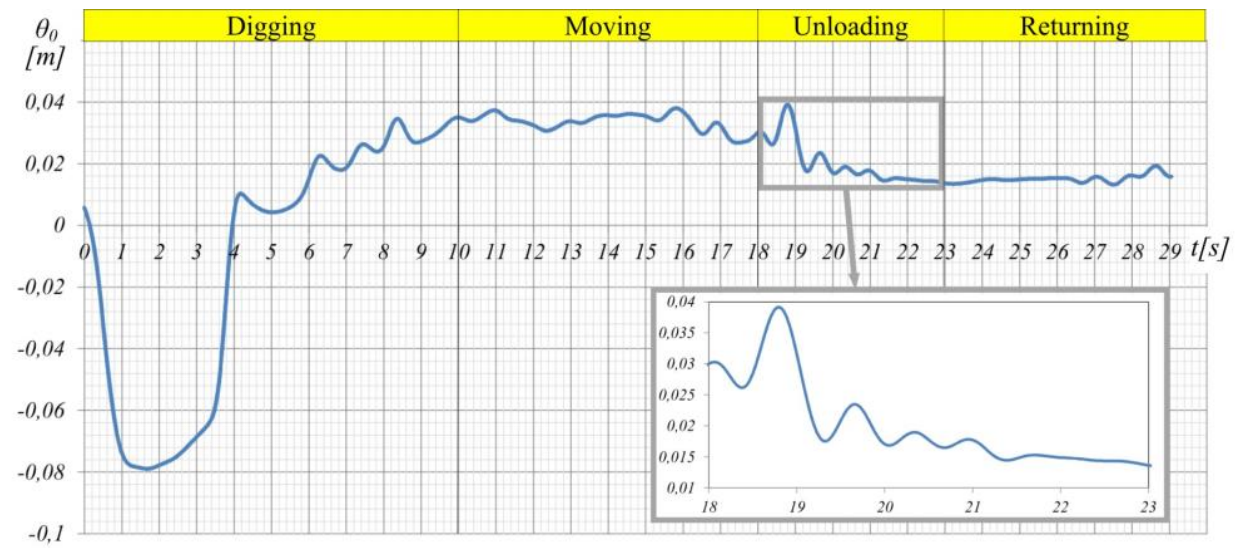

Fig. 6 The change in the vertical displacement of the support and movement member $\theta_{0}$ during the manipulation task of the excavator obtained in the experimental analysis

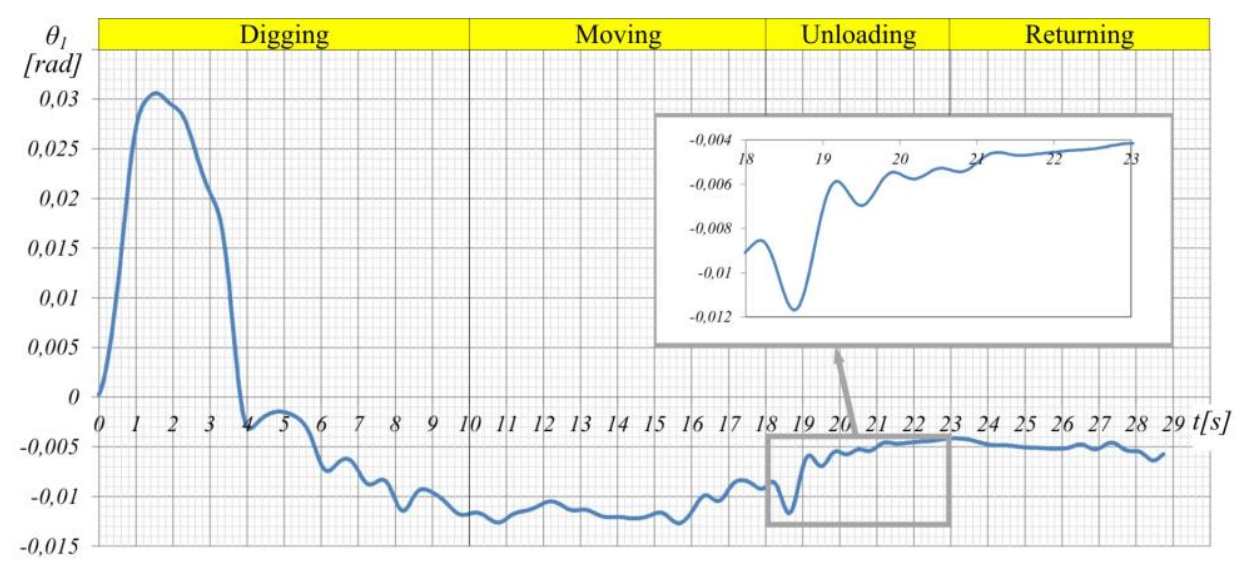

Fig. 7 The change in the angular displacement of the support and movement member $\theta_{1}$ during the manipulation task of the excavator obtained in the experimental analysis 


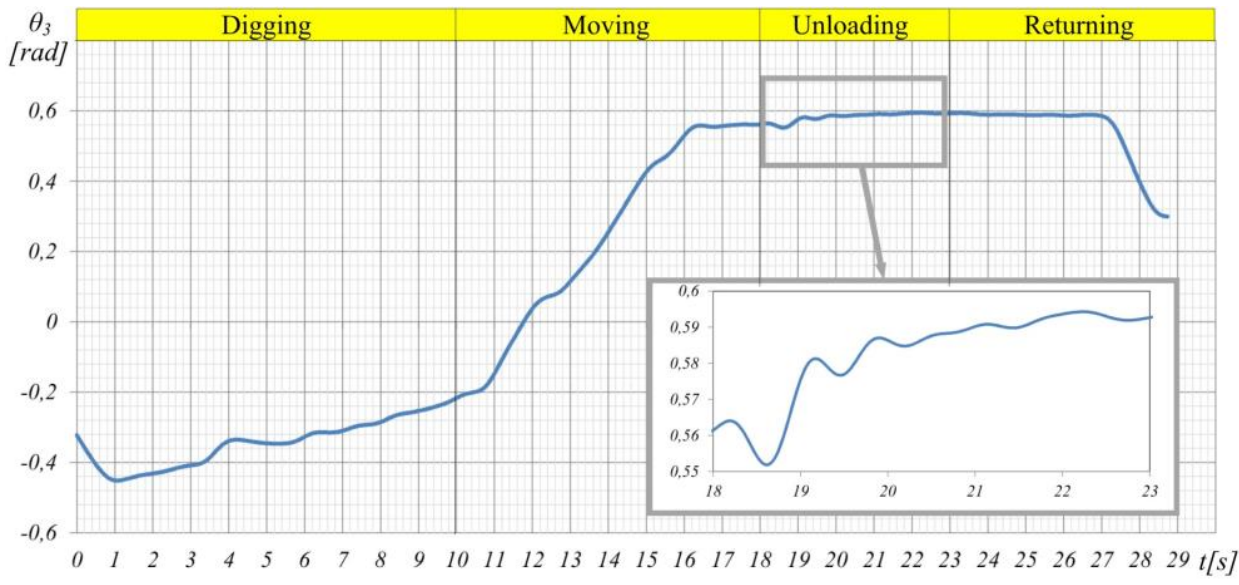

Fig. 8 The change in the vertical displacement of manipulator $\theta_{3}$ during the manipulation task of the excavator obtained in the experimental analysis

\section{CONCLUSION}

On the basis of the obtained results it can be concluded that the hydraulic excavators act like very sensitive dynamic oscillatory systems when performing manipulation tasks. Hydrostatic actuators (hydraulic motors and hydraulic cylinders) of drive mechanisms of the excavator kinematic chain appear as extremely elastically dampened system elements in the form of hydraulic springs caused by the hydraulic oil compressibility. Oil temperature in the excavator hydrostatic drive system affects the dynamic stability of the excavator. With an increase in oil temperature, amplitudes and periods of dampened oscillation of the excavator kinematic chain members also increase. The comparison of the character of changes and the duration of the calculated movement of the excavator, on the grounds of the defined mathematical model and the developed program, with the results obtained on the basis of the measured quantities of the excavator when operating in exploitation conditions show that the defined mathematical model possesses a sufficient accuracy to analyze the dynamic stability of the excavator.

Acknowledgements: The paper was prepared within the project TR 35049 financed by the Ministry of Education and Science of the Republic of Serbia.

\section{REFERENCES}

1. Nikolaevich, B.E., Nyrgaiazovich, B.I, Sergeevich Z.S., 2015, Enhancing the Stability of the Timber Harvesting Machine of Manipulator Type by Using an Active Suspension System, Journal of Applied Engineering Science, 13(2), pp. 111-116.

2. Ghasempoor, A., Sepehri, N., 1998, A Measure of Stability for Mobile Manipulators with Application to HeavyDuty Hydraulic Machines, Journal of Dynamic Systems, Measurement and Control, 120(3), pp. 360-370.

3. Abo-Shanab, R.F., Sepehri, N., 2004, Tip-Over Stability of Manipulator-Like Mobile Hydraulic Machines, Journal of Dynamic Systems, Measurement and Control, 127(2), pp. 295-301. 
4. Grigorov, B., Mitev, R., 2017, Dynamic behavior of a hydraulic crane operating a freely suspended paylood, Journal of Zhejiang University-SCIENCE A, 18(4), pp. 268-281.

5. Koivo, A. J., Thoma, M., Kocaoglan E., Andrade-Cetto, J., 1996, Modeling and Control of Excavator Dynamics during Digging Operation, Journal of Aerospace Engineering, 9(1), pp 10-18.

6. Dong, R., Pan, C., Hartsell, J., Welcome, D., Lutz, T., Brumfield, A., Harris, J., Wu, J., Wimer, B., Mucino V., Means, K., 2012, An Investigation on the Dynamic Stability of Scissor Lift, Open Journal of Safety Science and Technology, 2(1), pp. 8-15.

7. Mitrev, R., Janošević, D., Marinković, D., 2017, Dynamical Modelling of Hydraulic Excavator Considered as a Multibody System, Tehnicki Vjesnik (Technical Gazette), 24(Suppl. 2), pp. 327-338.

8. Jovanović, V., Janošević, D., Marinković, D., 2015, Determination of the Load Acting on the Axial Bearing of a Slewing Platform Drive in Hydraulic Excavators, Acta Polytechnica Hungarica, 12(1), pp. 5-22.

9. Dresig, H., Holzweißig, F., 2010, Dynamics of Machinery, Springer-Verlag Berlin Heidelberg.

10. Ewald, R., Hutter, J., Kretz, D., Liedhegener, F., Schenkel, W., Schmitt, A., Reik M., 1986, Proportional and Servo Valve Technology - Hydraulic trainer Vol. 2, Manesmann Rexroth.

11. Janošević, D., Jovanović, V., 2016, Synthesis of the drive mechanisms of a hydraulic excavator, Monograph, University of Niš, Faculty of Mechanical Engineering.

12. Muvenge, O.M., 2008, Simulation of the Dynamic Behavior of an Excavator due to Interacting Mechanical and Hydraulic Dynamics, Master thesis, Jomo Kenyatta University of Agriculture and Technology.

13. Fox, B., Jennings, L.S., Zomaya, A.Y., 2002, On the modeling of actuator dynamics and the computation of prescribed trajectories, Computers \& Structures, 80(7-8), pp. 605-614. 